\title{
Inhibitory effects of hydroxysafflor yellow A on PDGF-BB-induced proliferation and migration of vascular smooth muscle cells via mediating Akt signaling
}

\author{
YANMIN SONG, LILI LONG, NING ZHANG and YUNHAI LIU \\ Department of Neurology, Xiangya Hospital, Central South University, Changsha, Hunan 410008, P.R. China
}

Received November 20, 2013; Accepted May 29, 2014

DOI: $10.3892 / \mathrm{mmr} .2014 .2336$

\begin{abstract}
The abnormal proliferation and migration of vascular smooth muscle cells (VSMCs) are key pathological factors in the initiation and progression of vascular disorders, including arteriosclerosis and restenosis following percutaneous coronary intervention (PCI). Hydroxysafflor yellow A (HSYA), the main component of the safflower yellow pigments, has widely been used for the treatment of cardiovascular diseases in traditional Chinese medicine. However, to the best of our knowledge, there are no studies investigating the pharmaceutical effect of HSYA on VSMCs or the underlying molecular mechanism. The present study aimed to investigate the effect of HSYA on platelet-derived growth factor (PDGF)-BB-stimulated VSMC proliferation and migration. HSYA significantly inhibited PDGF-BB-stimulated VSMC proliferation and, in response to PDGF-BB-stimulation, VSMCs dedifferentiated into a proliferative phenotype. However, HSYA effectively reversed this phenotype switching. In addition, the production of nitrous oxide and cyclic guanosine monophosphate induced by PDGF-BB was also suppressed by HSYA, and HSYA markedly inhibited PDGF-BB-stimulated VSMC migration. Investigation of the molecular mechanism revealed that HSYA inhibited PDGF-BB-induced activation of Akt signaling. In addition, HSYA also suppressed PDGF-BB-stimulated upregulation of cell cycle related proteins and heme oxygenase-1. In conclusion, HSYA was able to inhibit PDGF-BB-stimulated VSMC proliferation and migration, partially via suppressing PDGF-BB-induced Akt signaling activation. Therefore, HSYA may be useful for the prevention and treatment of cardiovascular diseases, including atherosclerosis and restenosis following PCI.
\end{abstract}

Correspondence to: Professor Yunhai Liu, Department of Neurology, Xiangya Hospital, Central South University, 87 Xiangya Road, Changsha, Hunan 410008, P.R. China

E-mail: csuliuyunhai@163.com; csuliuyunhai@sina.com

Key words: hydroxysafflor yellow A, vascular smooth muscle cell, proliferation, migration, platelet-derived growth factor

\section{Introduction}

The abnormal proliferation and migration of vascular smooth muscle cells (VSMCs) in arterial walls is crucial in the initiation and progression of arteriosclerosis, as well as restenosis following percutaneous coronary intervention (PCI) or vein grafting (1). Thus, anti-proliferative and anti-migratory drugs for VSMCs are required for the prevention and treatment of vascular disorders.

Under physiological conditions, VSMCs remain in a quiescent state; however, in response to various stimuli, VSMCs switch to an uncontrolled proliferative and migratory state (2). For instance, following cardiovascular injury, including PCI or coronary artery bypass grafting, abundant cytokines and inflammatory factors are released. These released cytokines can initiate proliferative- and migratory-related signaling pathways, and markedly stimulate the proliferation and migration of VSMCs in arterial walls (3). Among cytokines, platelet-derived growth factor (PDGF)-BB has been demonstrated to have a critical role in vascular remodeling following vascular damage (4). During cellular and extracellular responses to vascular injury, the production of PDGF-BB is significantly increased, which further stimulates several key signaling pathways mediating VSMC proliferation and migration via binding to its receptor termed PDGFR $\beta$ (5). Therefore, the development of effective agents to inhibit PDGF-BB-stimulated VSMC proliferation and migration may be useful for the treatment of atherosclerosis and restenosis following PCI or coronary artery bypass grafting.

Hydroxysafflor yellow A (HSYA), the main component of the safflower yellow pigments, has been widely used for the treatment of cardiovascular diseases in traditional Chinese medicine (6). It has been suggested that HSYA has an inhibitory effect on platelet aggregation by antagonizing binding of the platelet activating factor to its receptor (7). In addition, HSYA has anti-hypotensive and anti-thrombotic effects $(8,9)$. However, to the best of our knowledge, there are no studies investigating the pharmaceutical effect of HSYA on VSMCs or the underlying molecular mechanism.

The present study aimed to determine the inhibitory effect of HSYA on PDGF-BB-stimulated VSMC proliferation and migration. In addition, the involved molecular mechanism was also investigated. 


\section{Materials and methods}

Materials and agents. HSYA was purchased from Lvye Natural Medicine Research and Development Center (Shandong, China). Recombinant human PDGF-BB was purchased from ProSpec-Tany TechnoGene (Rehovot, Israel). Dimethyl sulfoxide (DMSO) and MTT were purchased from Sigma-Aldrich (St. Louis, MO, USA). Mouse monoclonal anti-smooth muscle- $\alpha$-actin (SMA), mouse anti-desmin, mouse anti-smoothelin, mouse anti-phospho-Akt, mouse-anti-Akt, mouse anti-cyclin D1, mouse anti-cyclin E, mouse anti-cylcin-dependendent kinase 2 (CDK2), mouse anti-cyclin-dependent kinase 4 (CDK4) and mouse monoclonal anti-glyceraldehyde 3-phosphate dehydrogenase antibodies, as well as rabbit anti-mouse secondary antibody were obtained from Abcam (Cambridge, UK).

Cell culture. VSMCs were isolated from the thoracic aorta of 6- to 8-week-old male Sprague-Dawley rats. In the present study, all protocols were in accordance with the National Institutes of Health regulations for the care and use of animals in research (Bethesda, MA, USA) and were approved by the Ethics Committee of Central South University (Changsha, China). Isolated cells were cultured in Dulbecco's modified Eagle's medium (DMEM/F12; Invitrogen Life Technologies, Carlsbad, CA, USA) containing $10 \%$ fetal bovine serum (Invitrogen Life Technologies) at $37^{\circ} \mathrm{C}$ in a humidified atmosphere of $95 \%$ air and $5 \% \mathrm{CO}_{2}$. VSMCs at passage 4 were used in the following experiments.

VSMC proliferation assay. Prior to the assay, VSMCs were cultured to $70 \%$ confluence in a 96 -well plate. Subsequently, VSMCs in each well were serum-starved for $24 \mathrm{~h}$. Following this, in the control group, VSMCs were cultured without any treatment and in the PDGF-BB group, cells were treated with PDGF-BB $(20 \mathrm{ng} / \mathrm{ml})$ for $12,24,36$ and $48 \mathrm{~h}$. In the PDGF-BB + HSYA group, cells were treated with PDGF-BB $(20 \mathrm{ng} / \mathrm{ml})$ and HSYA $(20 \mu \mathrm{M})$ for $12,24,36$ and $48 \mathrm{~h}$. The effect of HSYA on PDGF-BB-induced VSMC proliferation was subsequently assayed using an MTT assay. In brief, the medium in each well was added with MTT at a final concentration of $0.5 \mu \mathrm{g} / \mathrm{ml}$. Following incubation for $3 \mathrm{~h}$, the medium was removed. DMSO (100 $\mu \mathrm{l})$ was added and the plate was gently rotated for $10 \mathrm{~min}$ to dissolve the precipitation. Cell proliferation was determined by measuring the absorbance at $550 \mathrm{~nm}$ using a microplate reader (Bio-Rad, Hercules, CA, USA).

Assay for nitrous oxide (NO) in VSMC medium. Following treatment with PDGF-BB $(20 \mathrm{ng} / \mathrm{ml})$ alone, or PDGF-BB $(20 \mathrm{ng} / \mathrm{ml})$ and HYSA $(20 \mu \mathrm{M})$ for $48 \mathrm{~h}$, the content of NO in the VSMC medium was determined using an NO enzyme immunoassay kit (Sigma-Aldrich), according to the manufacturer's instructions. VSMCs without treatment were used as the control group.

Assay for cyclic guanosine monophosphate (cGMP) in VSMCs. Following treatment with PDGF-BB $(20 \mathrm{ng} / \mathrm{ml})$ alone, or PDGF-BB $(20 \mathrm{ng} / \mathrm{ml})$ and HYSA $(20 \mu \mathrm{M})$ for $48 \mathrm{~h}$, the content of cGMP in VSMCs was determined using a cGMP enzyme immunoassay kit (GE Healthcare, Franklin Lakes, NJ, USA), according to the manufacturer's instructions. VSMCs without treatment were used as the control group. The total protein in each group was determined via a bicinchoninic acid assay (BCA) reaction (Pierce, Madison, WI, USA) and the data were normalized accordingly.

VSMC migration assay. VSMC migration was determined using a Transwell assay (BD Biosciences, Franklin Lake, NJ, USA), following treatment with PDGF-BB $(20 \mathrm{ng} / \mathrm{ml})$ alone, or PDGF-BB $(20 \mathrm{ng} / \mathrm{ml})$ and HYSA $(20 \mu \mathrm{M})$ for $48 \mathrm{~h}$. In brief, a 24-well modified Boyden chamber containing fibronectin-coated polycarbonate membranes (BD Biosciences) was used. For each group, the lower wells were filled with DMEM with or without PDGF-BB $(20 \mathrm{ng} / \mathrm{ml})$ in the presence or absence of HSYA $(20 \mu \mathrm{M})$ as indicated above. Following $24 \mathrm{~h}$ incubation at $37^{\circ} \mathrm{C}$ with $5 \% \mathrm{CO}_{2}$, cells on the upper side of the membrane were removed. Cells on the lower side of the membrane were stained with Hoechst 33342 (Beyotime, Shanghai, China) and counted in five randomly selected squares per well.

Western blotting assay. For the detection of protein expression in VSMCs in each group, western blotting was used. Briefly, VSMCs were lysed in radioimmunoprecipitation assay buffer (Beyotime), and the protein concentration was determined using a BCA Protein Assay kit (Thermo Fisher Scientific, Inc., Waltham, MA, USA). Subsequently, the protein was separated with 5\% SDS-PAGE and transferred onto a polyvinylidene difluoride membrane (Bio-Rad, Hercules, CA, USA), which was then blocked in 5\% nonfat dried milk in phosphate-buffered saline (Life Technologies) for $3 \mathrm{~h}$ at room temperature. Then, the membrane was incubated with specific primary antibodies for $3 \mathrm{~h}$. Subsequently, incubation with the appropriate secondary antibody and immune complexes were detected using an ECL kit (Pierce, Rockford, IL, USA).

Statistical analysis. All data are expressed as the mean \pm standard deviation of three independent experiments. Data were analyzed by one-way analysis of variance followed by Fisher's least significant difference post-hoc test. All analysis was performed using SPSS 17.0 software (SPSS, Inc., Chicago, IL, USA). $\mathrm{P}<0.05$ was considered to indicate a statistically significant difference.

\section{Results}

VSMC proliferation assays. An MTT assay was performed to determine the effect of HSYA on PDGF-BB-stimulated proliferation of VSMCs. The results demonstrated that VSMC proliferation was significantly lower in the experiment group compared with the control group and the NC group [VSMCs treated with only PDGF-BB (20 ng/ml) for 12, 24, 36 or $48 \mathrm{~h}$ ], suggesting that HSYA has a suppressive effect on the regulation of PDGF-BB-induced VSMC proliferation (Fig. 1).

VSMC phenotype assessments. As VSMCs can switch from a differentiated phenotype into a proliferative phenotype following vascular damage, western blotting was performed to determine the protein levels of SMA, smoothelin and desmin, three important markers for the differentiated phenotype of VSMCs. As 


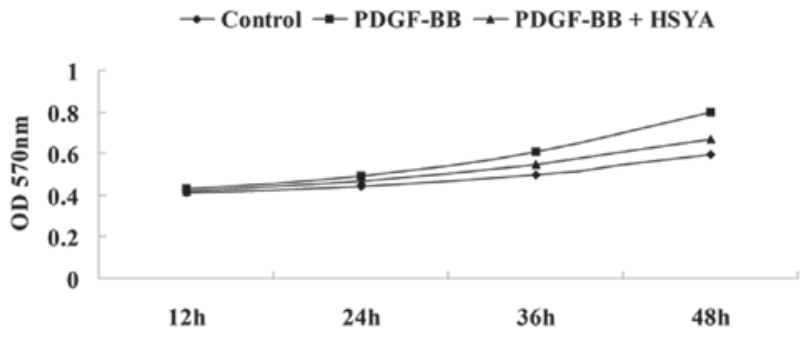

Figure 1. HSYA inhibits PDGF-BB-stimulated VSMC proliferation. An MTT assay was used to examine the effect of HYSA and PDGF-BB on the proliferation of VSMCs. Control: VSMCs were cultured without any treatment. PDGF-BB: VSMCs were treated with only PDGF-BB $(20 \mathrm{ng} / \mathrm{ml})$ for 12,24 , 36 and $48 \mathrm{~h}$. PDGF-BB + HSYA: VSMCs were treated with HSYA $(20 \mu \mathrm{M})$ and PDGF-BB $(20 \mathrm{ng} / \mathrm{ml})$ for 12, 24, 36 and $48 \mathrm{~h}$. HYSA, hydroxysafflor yellow A; PDGF-BB, platelet derived growth factor BB; VSMCs, vascular smooth muscle cells; OD, optical density.
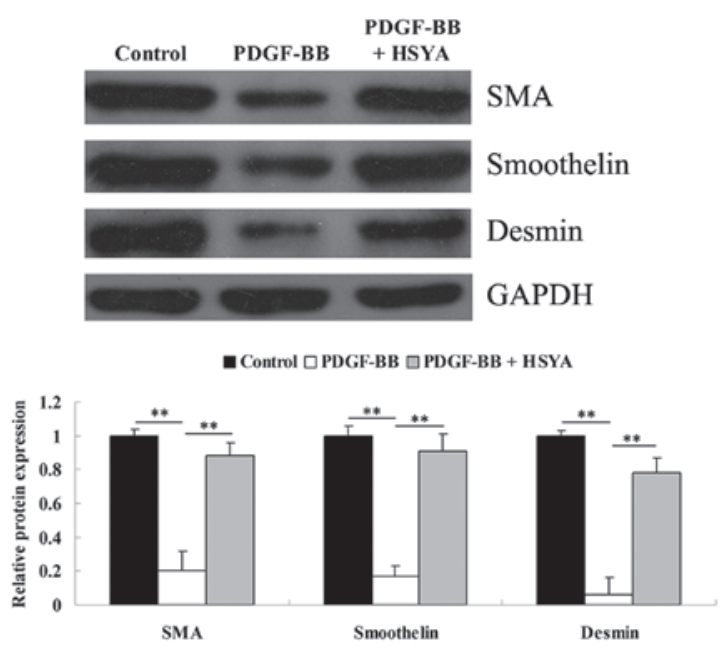

Figure 2. HSYA inhibits PDGF-BB-induced phenotype switching of VSMCs. The protein expression of smooth muscle markers SMA, smoothelin and desmin were determined by western blotting. Control: VSMCs were cultured without any treatment. PDGF-BB: VSMCs were treated with only PDGF-BB $(20 \mathrm{ng} / \mathrm{ml})$ for $48 \mathrm{~h}$. PDGF-BB + HSYA: VSMCs were treated with HSYA $(20 \mu \mathrm{M})$ and PDGF-BB $(20 \mathrm{ng} / \mathrm{ml})$ for $48 \mathrm{~h} .{ }^{* *} \mathrm{P}<0.01$. HYSA, hydroxysafflor yellow A; PDGF-BB, platelet derived growth factor BB; VSMCs, vascular smooth muscle cells; SMA, smooth muscle actin; GAPDH, glyceraldehyde 3-phosphate dehydrogenase.

shown in Fig. 2, incubation with PDGF-BB for $48 \mathrm{~h}$ significantly downregulated the protein levels of SMA, smoothelin and desmin in VSMCs, suggesting that VSMCs dedifferentiate into a proliferative phenotype. However, in the experiment group, the expression of these three markers remained high, indicating that HSYA was able to suppress the PDGF-BB-induced switch of VSMCs into a proliferative phenotype.

Alterations in NO content in the VSMC medium and cGMP production in VSMCs. The present study also determined the cGMP level in VSMCs and the NO content in the supernatant of each group. As demonstrated in Fig. 3A, the cGMP level in VSMCs was reduced by incubation with PDGF-BB for $48 \mathrm{~h}$, which was inhibited by addition of HSYA. Consistent with the changes in cGMP level in VSMCs, PDGF-BB also markedly downregulated the NO content in the supernatant of VSMCs, which was effectively reversed by HSYA (Fig. 3B).

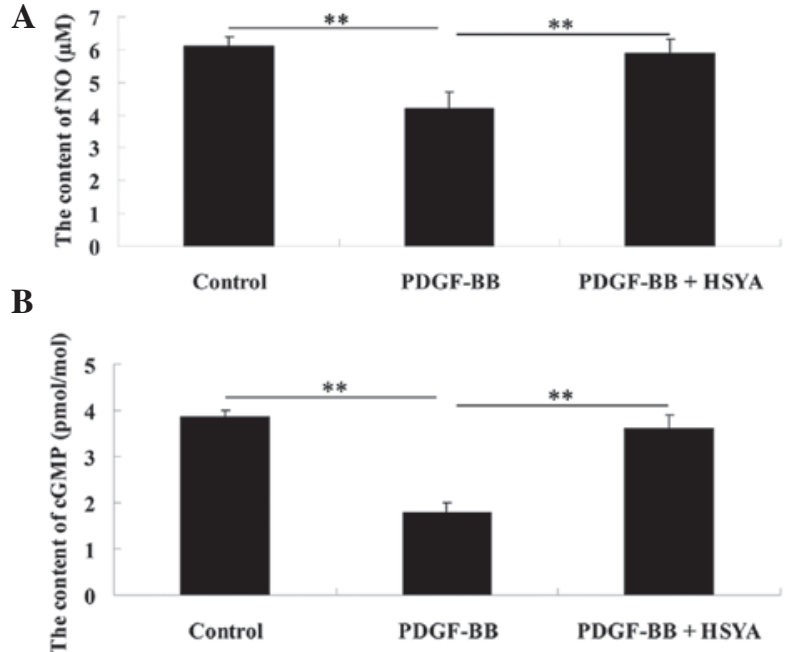

Figure 3. HSYA suppresses PDGF-BB-stimulated downregulation of NO and cGMP production in VSMCs. The NO content in the medium and the cGMP level in VSMCs were determined. Control: VSMCs were cultured without any treatment. PDGF-BB: VSMCs were treated with only PDGF-BB (20 $\mathrm{ng} / \mathrm{ml})$ for $48 \mathrm{~h}$. PDGF-BB + HSYA: VSMCs were treated with HSYA $(20 \mu \mathrm{M})$ and PDGF-BB $(20 \mathrm{ng} / \mathrm{ml})$ for $48 \mathrm{~h} .{ }^{* *} \mathrm{P}<0.01$. HYSA, hydroxysafflor yellow A; PDGF-BB, platelet derived growth factor BB; NO, nitrous oxide; cGMP, cyclic guanosine monophosphate; VSMCs, vascular smooth muscle cells.

VSMC migration assays. The effect of HSYA on PDGF-BB-stimulated VSMC migration was investigated by performing a Transwell assay. As shown in Fig. 4, stimulation of PDGF-BB for $48 \mathrm{~h}$ significantly promoted VSMC migration compared with the control group; however, HSYA effectively inhibited the stimulatory effect of PDGF-BB on VSMC migration.

Alterations in Akt signaling activity. Akt signaling is important in VSMC proliferation in response to inflammation and oxidative stress (10). Therefore, the present study examined the activity of Akt signaling in VSMCs stimulated by PDGF-BB, in the presence or absence of HSYA, using western blotting. The results demonstrated that the phospho-Akt level in PDGF-BB-stimulated VSMCs was significantly upregulated, compared with that in VSMCs without treatment. However, HSYA significantly inhibited the upregulation of phospho-Akt in PDGF-BB-stimulated VSMCs. These findings suggest that the suppressive effect of HSYA on PDGF-BB-stimulated VSMC proliferation is at least partially through inhibition of Akt signaling activation (Fig. 5).

Alterations in cell cycle related protein expression. Furthermore, as Akt signaling is involved in the regulation of cell cycle progression by controlling cyclins and CDKs (11), the expression levels of cyclin D1, cyclin E, CDK2 and CDK4 were investigated. As demonstrated in Fig. 6, HSYA inhibited the PDGF-BB-induced upregulation of cyclin D1, cyclin E, $\mathrm{CDK} 2$ and $\mathrm{CDK} 4$ protein expression.

Alterations in the protein expression of heme oxygenase-1 (HO-1). HO-1 has been demonstrated to have a suppressive effect in PDGF-BB-induced VSMC proliferation and migration $(12,13)$. Therefore, the present study determined the 

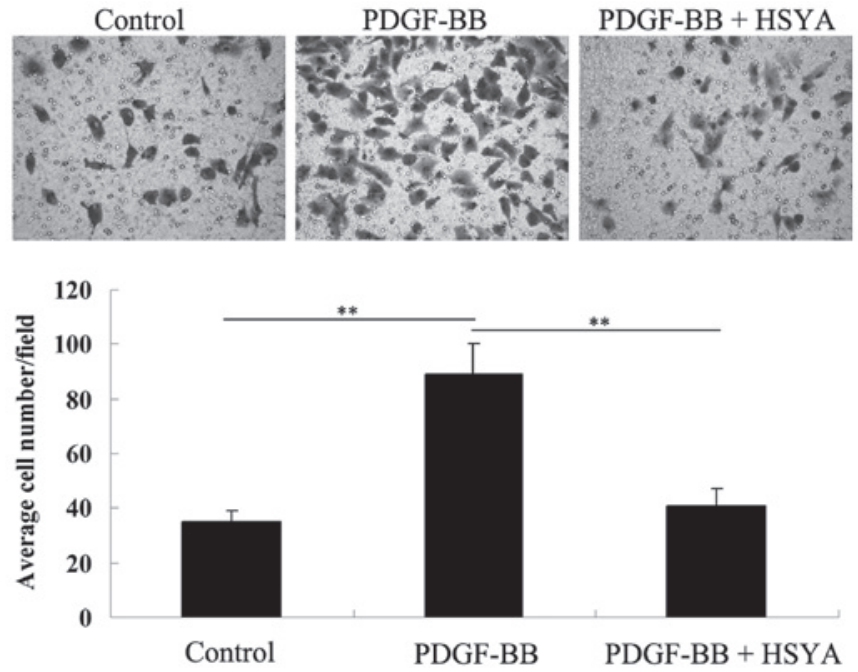

Figure 4. HSYA suppresses PDGF-BB-stimulated VSMC migration. A transwell assay was used to determine the effects of HSYA and PDGF-BB on the migration of VSMCs. Control: VSMCs were cultured without any treatment. PDGF-BB: VSMCs were treated with only PDGF-BB $(20 \mathrm{ng} / \mathrm{ml})$ for 48 h. PDGF-BB + HSYA: VSMCs were treated with HSYA $(20 \mu \mathrm{M})$ and PDGF-BB $(20 \mathrm{ng} / \mathrm{ml})$ for $48 \mathrm{~h}$. The cell number was counted in five randomly selected fields. ${ }^{* *} \mathrm{P}<0.01$. HYSA, hydroxysafflor yellow A; PDGF-BB, platelet derived growth factor BB; VSMCs, vascular smooth muscle cells.

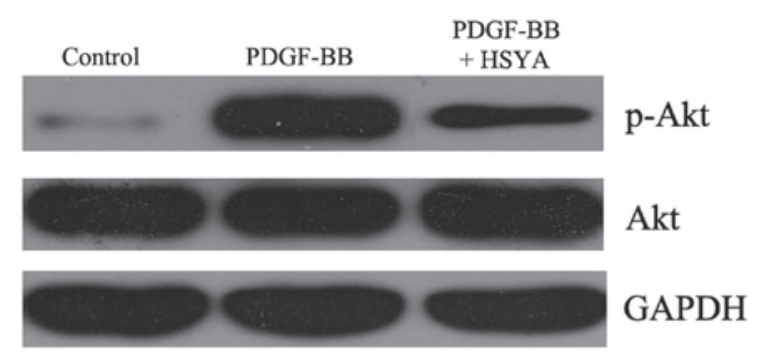

Figure 5. HSYA suppresses PDGF-BB-stimulated activation of Akt signaling in VSMCs. Western blotting was used to determine the activity of Akt signaling in each group. Control: VSMCs were cultured without any treatment. PDGF-BB: VSMCs were treated only with PDGF-BB $(20 \mathrm{ng} / \mathrm{ml})$ for $48 \mathrm{~h}$. PDGF-BB + HSYA: VSMCs were treated with HSYA $(20 \mu \mathrm{M})$ and PDGF-BB $(20 \mathrm{ng} / \mathrm{ml})$ for $48 \mathrm{~h}$. HYSA, hydroxysafflor yellow A; PDGF-BB, platelet derived growth factor BB; VSMCs, vascular smooth muscle cells; GADPH, glyceraldehyde 3-phosphate dehydrogenase.

protein expression of HO-1 in each group. As shown in Fig. 7, HSYA significantly inhibited PDGF-BB-stimulated downregulation of HO-1 protein expression.

\section{Discussion}

Carthamus tinctorius L., the flower of the safflower-plant, has been widely used in the treatment of cerebrovascular and cardiovascular disease in traditional Chinese medicine. Its extracts contain yellow and red pigments, including safflomin A, safflomin C and safflor yellow B, as well as HSYA, which has been demonstrated to be the most active chemical component $(14,15)$. Previous evidence has suggested that HSYA has a protective effect on cardiovascular disease $(15,16)$. For instance, Nie et al reported that HSYA was able to significantly reduce blood pressure and heart rate, possibly through the activation of BK (Ca) and K (ATP) channels (17). HSYA was also reported to inhibit auto-antibody

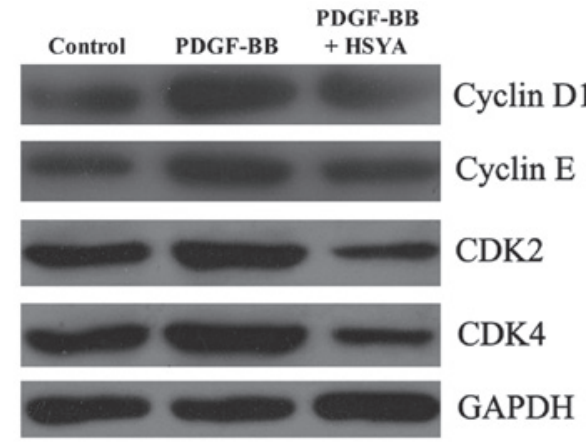

a Control $\square$ PDGF-BB $\square$ PDGF-BB + HSYA

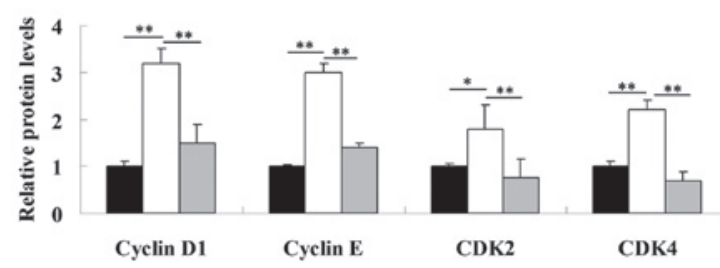

Figure 6. HSYA suppresses PDGF-BB stimulated upregulation of cell cycle related protein levels in VSMCs. Western blotting was used to determine the levels of cell cycle related proteins, including cyclin D1, cyclin E, CDK2 and CDK4 in each group. Control: VSMCs were cultured without any treatment. PDGF-BB: VSMCs were treated with only PDGF-BB $(20 \mathrm{ng} / \mathrm{ml})$ for 48 h. PDGF-BB + HSYA: VSMCs were treated with HSYA $(20 \mu \mathrm{M})$ and PDGF-BB $(20 \mathrm{ng} / \mathrm{ml})$ for $48 \mathrm{~h} .{ }^{*} \mathrm{P}<0.05,{ }^{* *} \mathrm{P}<0.01$. HYSA, hydroxysafflor yellow A; PDGF-BB, platelet derived growth factor BB; VSMCs, vascular smooth muscle cells; CDK, cyclin-dependent kinase; GADPH, glyceraldehyde 3-phosphate dehydrogenase.
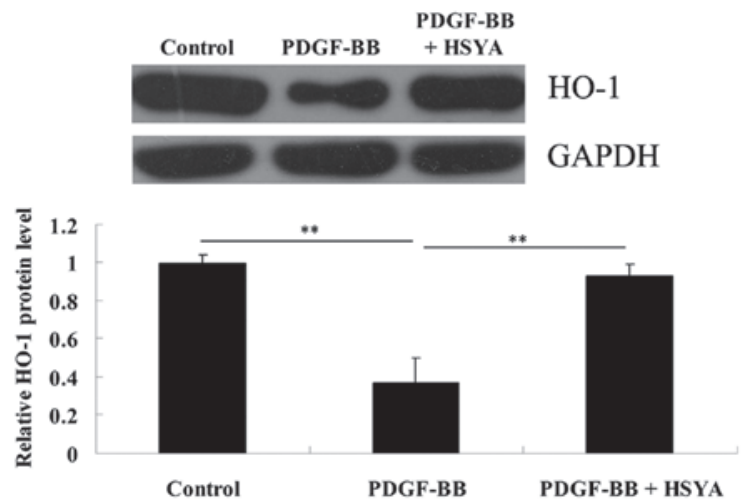

Figure 7. HSYA suppresses PDGF-BB-induced downregulation of HO-1 protein expression in VSMCs. Western blotting was used to determine the protein level of HO-1 in each group. Control: VSMCs were cultured without any treatment. PDGF-BB: VSMCs were treated with only PDGF-BB $(20 \mathrm{ng} / \mathrm{ml})$ for $48 \mathrm{~h}$. PDGF-BB + HSYA: VSMCs were treated with HSYA $(20 \mu \mathrm{M})$ and PDGF-BB $(20 \mathrm{ng} / \mathrm{ml})$ for $48 \mathrm{~h} .{ }^{* *} \mathrm{P}<0.01$. HYSA, hydroxysafflor yellow A; PDGF-BB, platelet derived growth factor BB; HO-1, heme oxygenase-1; VSMCs, vascular smooth muscle cells; GADPH, glyceraldehyde 3-phosphate dehydrogenase.

against AT1 receptor-induced vascular endothelial cell injury and VSMC proliferation in vivo, indicating that HSYA has protective effects on vascular endothelial cells and the function of VSMCs (18). However, to the best of our knowledge, the underlying molecular mechanism of HSYA in cytokine-stimulated VSMC proliferation and migration has not been previously investigated. The present study demonstrated for the first time, to the best of our knowledge, that HSYA effectively inhibited PDGF-BB-induced VSMC proliferation 
and migration, dedifferentiation into a proliferative phenotype, activation of AKT activity and upregulation of cell cycle related proteins.

Following vascular injury, upregulated production of inflammatory factors and cytokines promotes the proliferation and migration of VSMCs, leading to neointima formation (19). It has been demonstrated that neointima formation is important in various cardiovascular diseases, including hypertension, atherosclerosis and restenosis following PCI $(20,21)$. Therefore, inhibition of neointima formation by suppressing cytokine-stimulated VSMC proliferation and migration is an effective strategy for the prevention and treatment of cardiovascular disorders. The present study reported that HSYA inhibited PDGF-BB-stimulated VSMC proliferation. Since vascular injury was able to induce VSMCs to dedifferentiate into a proliferative phenotype, the present study investigated the effect of HSYA on the PDGF-BB-induced phenotype switch of VSMCs. The results demonstrated that PDGF-BB treatment markedly inhibited the expression levels of smooth muscle markers, including SMA, smoothelin and desmin, indicating that VSMCs dedifferentiated into a proliferative phenotype. However, HSYA effectively restored their expression, suggesting that HSYA maintained the differentiated phenotype of VSMCs, and thus suppressed PDGF-BB-stimulated VSMC proliferation. In addition, the upregulated expression of cell cycle proteins induced by PDGF-BB treatment was also inhibited by HSYA in VSMCs, suggesting that cell cycle progression was suppressed.

NO has been found to have an inhibitory effect on the regulation of VSMC proliferation (22). The present study demonstrated that HSYA significantly inhibited PDGF-BB-induced downregulation of NO production. Furthermore, it is well established that NO can stimulate the formation of cGMP, which also has a suppressive effect on the proliferation of VSMCs (23). Therefore, the present study examined the level of cGMP in VSMCs, and demonstrated that HSYA significantly inhibited PDGF-BB-stimulated cGMP formation. These findings suggest that HSYA suppresses PDGF-BB-induced VSMC proliferation, possibly through mediating NO/cGMP-dependent mechanisms.

VSMC migration has also been demonstrated to be important in the initial step of neointima formation, and thus is closely associated with the development of atherosclerotic lesions and restenosis following PCI $(24,25)$. Therefore, the present study investigated the effect of HSYA on VSMC migration following incubation with PDGF-BB for $48 \mathrm{~h}$. The results demonstrated that HSYA effectively reversed PDGF-BB-stimulated VSMC migration.

Inflammatory responses have been demonstrated to act as a key pathogenic factor in cardiovascular diseases (26). Following stimulation by inflammatory cytokines, the AKT signaling pathway is activated, leading to the upregulated proliferation and migration of VSMCs (12). Furthermore, it is also well established that PDGF-BB can stimulate VSMC proliferation and migration via activation of the Akt signaling pathway (10). In addition, Akt has been demonstrated to have a key regulatory role in vascular remodeling (27). Therefore, the activity of AKT signaling was further determined. Data from the present study revealed that HSYA inhibited PDGF-BB-induced Akt signaling activation, indicating that the inhibitory role of HSYA in PDGF-BB-induced VSMC proliferation and migration is possibly through its repressive effect on the activation of the Akt signaling pathway.

$\mathrm{HO}-1$, a rate-limiting enzyme in the degradation of heme (a potent oxidant), is highly inducible by heme as well as other substances, including hydrogen peroxide and endotoxin (28). Furthermore, HO-1 has been demonstrated to be highly expressed in vascular tissues and have intracellular anti-inflammatory, anti-oxidant and anti-apoptotic effects (29). Previous studies have suggested that HO-1 may have a protective effect on the vascular system. Jiang et al reported that $\mathrm{HO}-1$ protected VSMCs from oxidative injury (12). Cheng et al demonstrated that HO-1 antagonized abnormal proliferation and migration of VSMCs induced by PDGF-BB (13). In addition, the beneficial effect of HO-1 on atherosclerosis has also been reported (30). The present study demonstrated that, following treatment of PDGF-BB, the protein level of HO-1 was significantly downregulated, accompanied by increased proliferation and migration; however, HSYA markedly restored the expression of HO-1. Based on these findings, it was suggested that HSYA is able to protect against PDGF-BB-induced HO-1 downregulation.

In conclusion, the present study, for the first time, to the best of our knowledge, demonstrated that HSYA inhibited PDGF-BB-induced VSMC proliferation and migration, possibly through its inhibitory effects on PDGF-BB-stimulated Akt signaling activation, as well as cell cycle related proteins and the expression of HO-1 in VSMCs. Therefore, HSYA may be a promising agent for the prevention and treatment of arteriosclerosis and restenosis following PCI via inhibition of neointima formation, an important step in vascular lesion formation.

\section{Acknowledgements}

This study was supported by the Science and Technology Planning of Hunan Province (No. 2013SK5017), Natural Science Foundation of Hunan Province, China (No. 13JJ5005) and National Natural Science Foundation of China (No. 81201001).

\section{References}

1. Gan J,Li P, Wang Z, et al: Rosuvastatin suppresses platelet-derived growth factor-BB-induced vascular smooth muscle cell proliferation and migration via the MAPK signaling pathway. Exp Ther Med 6: 899-903, 2013.

2. Chen YC, Chu LY, Yang SF, et al: Prostacyclin and PPARalpha agonists control vascular smooth muscle cell apoptosis and phenotypic switch through distinct 14-3-3 isoforms. PLoS One 8: e69702, 2013.

3. Hakimi M, Peters A, Becker A, Bockler D and Dihlmann S: Inflammation-related induction of absent in melanoma 2 (AIM2) in vascular cells and atherosclerotic lesions suggests a role in vascular pathogenesis. J Vasc Surg 5: 794-803, 2013

4. Li P, Liu Y, Yi B, et al: MicroRNA-638 is highly expressed in human vascular smooth muscle cells and inhibits PDGF-BB-induced cell proliferation and migration through targeting orphan nuclear receptor NOR1. Cardiovasc Res 99: 185-193, 2013.

5. Li QL, Gu FM, Wang Z, et al: Activation of PI3K/AKT and MAPK pathway through a PDGFRbeta-dependent feedback loop is involved in rapamycin resistance in hepatocellular carcinoma. PLoS One 7: e33379, 2012.

6. Wang J, Zhang Q, Mei X and Zhang X: Hydroxysafflor yellow A attenuates left ventricular remodeling after pressure overload-induced cardiac hypertrophy in rats. Pharm Biol 52: 31-35, 2013. 
7. Zang BX, Jin M, Si N, et al: Antagonistic effect of hydroxysafflor yellow A on the platelet activating factor receptor. Yao Xue Xue Bao 37: 696-699, 2002 (In Chinese).

8. Liu L, Duan JA, Tang Y, et al: Taoren-Honghua herb pair and its main components promoting blood circulation through influencing on hemorheology, plasma coagulation and platelet aggregation. J Ethnopharmacol 139: 381-387, 2012.

9. Zhu HB, Zhang L, Wang ZH, et al: Therapeutic effects of hydroxysafflor yellow A on focal cerebral ischemic injury in rats and its primary mechanisms. J Asian Nat Prod Res 7: 607-613, 2005.

10. Park ES, Kang SI, Yoo KD, et al: Camptothecin inhibits platelet-derived growth factor-BB-induced proliferation of rat aortic vascular smooth muscle cells through inhibition of PI3K/Akt signaling pathway. Exp Cell Res 319: 982-991, 2013.

11. Jin YJ, Lee JH, Kim YM, Oh GT and Lee H: Macrophage inhibitory cy tokine-1 stimulates proliferation of human umbilica vein endothelial cells by up-regulating cyclins D1 and E through the PI3K/Akt-, ERK-, and JNK-dependent AP-1 and E2F activation signaling pathways. Cell Signal 24: 1485-1495, 2012.

12. Jiang F, Jiang R, Zhu X, Zhang X and Zhan Z: Genipin inhibits TNF-alpha-induced vascular smooth muscle cell proliferation and migration via induction of HO-1. PLoS One 8: e74826, 2013.

13. Cheng C, Haasdijk RA, Tempel D, et al: PDGF-induced migration of vascular smooth muscle cells is inhibited by heme oxygenase-1 via VEGFR2 upregulation and subsequent assembly of inactive VEGFR2/PDGFRbeta heterodimers. Arterioscler Thromb Vasc Biol 32: 1289-1298, 2012.

14. Bai Y, Lu P, Han C, et al: Hydroxysafflor yellow A (HSYA) from flowers of Carthamus tinctorius L. and its vasodilatation effects on pulmonary artery. Molecules 17: 14918-14927, 2012.

15. Han SY, Li HX, Ma X, et al: Evaluation of the anti-myocardial ischemia effect of individual and combined extracts of Panax notoginseng and Carthamus tinctorius in rats. J Ethnopharmacol 145: 722-727, 2013.

16. Wan LH, Chen J, Li L, Xiong WB and Zhou LM: Protective effects of Carthamus tinctorius injection on isoprenaline-induced myocardial injury in rats. Pharm Biol 49: 1204-1209, 2011

17. Nie PH, Zhang L, Zhang WH, Rong WF and Zhi JM: The effects of hydroxysafflor yellow A on blood pressure and cardiac function. J Ethnopharmacol 139: 746-750, 2012.

18. Jin Z, Zhang W, Chai W, Zheng Y and Zhi J: Antibodies against AT1 receptors are associated with vascular endothelial and smooth muscle function impairment: protective effects of hydroxysafflor yellow A. PLoS One 8: e67020, 2013.
19. Xiao Q, Zhang F, Grassia G, et al: Matrix metalloproteinase-8 promotes vascular smooth muscle cell proliferation and neointima formation. Arterioscler Thromb Vasc Biol 35: 90-98, 2014.

20. Stansfield BK, Bessler WK, Mali R, et al: Ras-Mek-Erk signaling regulates $\mathrm{Nf} 1$ heterozygous neointima formation. Am J Pathol 184: 79-85, 2014.

21. O' Brien ER, Ma X, Simard T, Pourdjabbar A and Hibbert B: Pathogenesis of neointima formation following vascular injury. Cardiovasc Hematol Disord Drug Targets 11: 30-39, 2011.

22. Guo J, Li L, Wu YJ, et al: Inhibitory effects of Brazilin on the vascular smooth muscle cell proliferation and migration induced by PDGF-BB. Am J Chin Med 41: 1283-1296, 2013.

23. Hwang SM, Lee YJ, Lee YP, et al: Anti-proliferative effect of an aqueous extract of Prunella vulgaris in vascular smooth muscle cells. Evid Based Complement Alternat Med 2013: 936463 , 2013.

24. Sartore S, Chiavegato A, Faggin E, et al: Contribution of adventitial fibroblasts to neointima formation and vascular remodeling: from innocent bystander to active participant. Circ Res 89: $1111-1121,2001$

25. Karki R, Kim SB and Kim DW: Magnolol inhibits migration of vascular smooth muscle cells via cytoskeletal remodeling pathway to attenuate neointima formation. Exp Cell Res 319: 3238-3250, 2013.

26. Lowe G, Woodward M, Hillis G, et al: Circulating inflammatory markers and the risk of vascular complications and mortality in people with Type 2 Diabetes mellitus and cardiovascular disease or risk factors: The ADVANCE study. Diabetes 63: 1115-1123, 2013.

27. Sedding DG, Widmer-Teske R, Mueller A, et al: Role of the phosphatase PTEN in early vascular remodeling. PLoS One 8: e55445, 2013.

28. Son Y, Lee JH, Chung HT and Pae HO: Therapeutic roles of heme oxygenase- 1 in metabolic diseases: curcumin and resveratrol analogues as possible inducers of heme oxygenase-1. Oxid Med Cell Longev 2013: 639541, 2013.

29. Wu ML, Ho YC and Yet SF: A central role of heme oxygenase-1 in cardiovascular protection. Antioxid Redox Signal 15: 1835-1846, 2011.

30. Yang HL, Chang HC, Lin SW, et al: Antrodia salmonea inhibits TNF-alpha-induced angiogenesis and atherogenesis in human endothelial cells through the down-regulation of NF-kappaB and up-regulation of Nrf2 signaling pathways. J Ethnopharmacol 15: 394-406, 2014 